Review

\title{
Antitumor functions and mechanisms of nitidine chloride in human cancers
}

\author{
Yue Cui ${ }^{1}$, Linhui $\mathrm{Wu}^{1}$, Ruoxue $\mathrm{Cao}^{1}$, Hui $\mathrm{Xu}^{2}$, Jun $\mathrm{Xia}^{3}{ }^{\bowtie}, \mathrm{Z}$ Peter Wang ${ }^{3,4}{ }^{\bowtie}$, Jia $\mathrm{Ma}^{3 凶}$ \\ 1. Research Center of Clinical Laboratory Science, School of Laboratory Medicine, Bengbu Medical College, Anhui, China, 233030, China. \\ 2. Department of Laboratory Medicine, School of Laboratory Medicine, Bengbu Medical College, Anhui, 233030, China. \\ 3. Department of Biochemistry and Molecular Biology, School of Laboratory Medicine, Bengbu Medical College, Anhui, 233030, China. \\ 4. Department of Pathology, Beth Israel Deaconess Medical Center, Harvard Medical School, Boston, MA, USA. \\ $\square$ Corresponding authors: Jun Xia, Z Peter Wang, and Jia Ma. Department of Biochemistry and Molecular Biology, Bengbu Medical College, 2600 Donghai \\ Street, Anhui, 233030, China. Email: xiajunbbmu@126.com (JX); majiamj10@126.com (JM); zwang6@bidmc.harvard.edu (ZPW).
}

(c) The author(s). This is an open access article distributed under the terms of the Creative Commons Attribution License (https://creativecommons.org/licenses/by/4.0/). See http://ivyspring.com/terms for full terms and conditions.

Received: 2019.06.25; Accepted: 2019.11.11; Published: 2020.01.01

\begin{abstract}
Nitidine chloride (NC), a quaternary ammonium alkaloid, exhibits multiple biological activities, including antimalarial, antifungal, and antiangiogenesis. Recently, NC has been characterized to perform antitumor activity in a variety of malignancies. NC has been identified to suppress cell proliferation, stimulate apoptosis, and induce cell cycle arrest, retard migration, invasion and metastasis. Moreover, NC is reported to sensitize cancer cells to chemotherapeutic drugs. In this review article, we describe the functions of $\mathrm{NC}$ in human cancers and discuss the molecular insight into NC-involved antitumor feature. This review article will stimulate the deeper investigation for using $\mathrm{NC}$ as a potent agent for the management of cancer patients.
\end{abstract}

Key words: Nitidine chloride; Cancer; Therapy; Target; Antitumor

\section{Introduction}

Nitidine, a phytochemical alkaloid, is mainly extracted from the root of Zanthoxylum nitidum that belongs to the family of Rutaceae, which is often distributed in Northeastern Asia [1] (Figure 1). Nitidine has been characterized as a folk medicine because it is helpful for reducing pain, enhancing blood circulation and cleaning blood stasis [1]. Some studies have revealed that nitidine has effects on protection of tumor, inflammation, and HIV infection $[2,3]$. NC exhibits multiple biological activities including antimalarial [4], antifungal [5], and antiangiogenesis [6]. Moreover, NC has been identified to inhibit cell proliferation, induce apoptosis, trigger cell cycle arrest, and sensitize cancer cells to chemotherapeutic drugs. Some researchers have discovered the underlying antitumor mechanisms of NC in human cancers $[1,7]$. In this review article, we describe the functions of NC in human cancers and highlight the molecular insight of NC-induced antitumor feature (Figure 2).

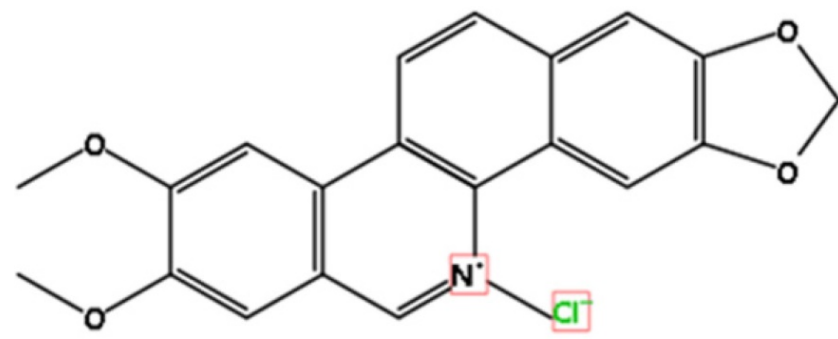

Figure 1. The structure of Nitidine chloride is illustrated. The molecular formula is C21H18CLNO4.

\section{Role of NC in human cancers}

\section{Breast cancer}

Breast cancer is one of the leading causes of cancer-mediated deaths in female [8]. Currently, the treatments of breast cancer include surgery, radiotherapy, and chemotherapy. However, due to metastasis and drug resistance, breast cancer patients have poor prognosis [8]. NC has been reported to 


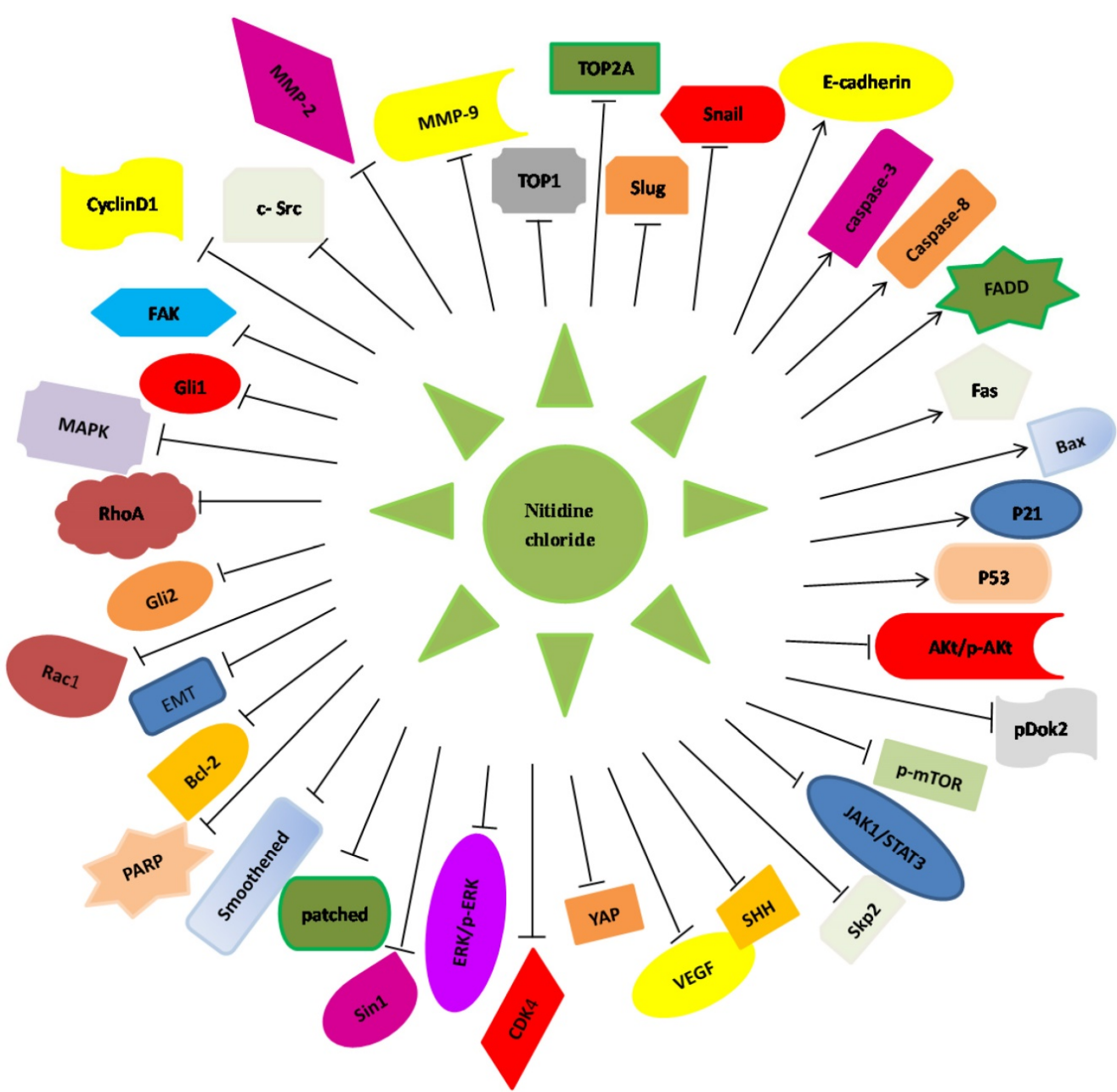

Figure 2. Illustration of Nitidine chloride -regulated targets and signaling pathways in human cancers. Nitidine chloride exerts its anti-tumor function via regulating the expression of these downstream genes in human malignancies.

inhibit the metastasis of breast cancer cells via inactivation of the c-Src/FAK-associated pathway [3]. Moreover, NC decreased the MMP-9 and MMP-2 formation and their proteolytic activity in mammary cancer cells [3]. Mechanistically, NC reduced PDGF-triggered phosphorylation of c-Src, FAK, MAPK, and inactivated the activity of RhoA, Rac1, and AP [3]. Another study revealed that NC exposure led to inhibition of cell proliferation and induction of cell cycle arrest through elevation of multiple gene expressions such as p53, p21, Bax, and active forms of caspase-3, caspase-9, and cleaved PARP, and downregulation of Bcl-2 [9]. Notably, NC sensitizes cell sensitivity to doxorubicin for cell proliferation in breast cancer [9].

Accumulating evidence suggest that EMT plays an essential role in tumor metastasis due to that mesenchymal cells acquire more migratory feature [8]. EMT is a programme that epithelial cells transfer to mesenchymal cells, which is often happened in multiple biological processes such as fibrosis, wound healing, and tumor metastasis [10]. The cell phenotype is changed from apical-basal polarity and tight junctions to elongated and spindle-shape cells and loose interaction, leading to increased migration and invasion [11]. EMT molecular markers are changed from loss of epithelial markers such as E-cadherin to acquired mesenchymal molecules such as Slug, Snail, Vimentin, Zeb1, and Zeb2 [12]. Various EMT inducers led to EMT in cells, which have CSC characters [13, 14]. CSC have been identified in multiple types of cancers, which is associated with tumor metastasis, drug resistance, and tumor reoccurrence [15]. CD44+/CD24- has been characterized as a marker for breast CSC, and CSC are involved in tumor metastasis and radiotherapy resistance [16, 17]. Recently, Sun et al. identified that NC inhibited migratory and invasive capability due to suppression of EMT and CSC-like phenotype by suppression of $\mathrm{HH}$ pathway in breast cancer cells [18]. Specifically, NC downregulated the expression of several molecules in $\mathrm{HH}$ pathway including Gli1 and Gli2, suppressed the expression level of mesenchymal markers such as Zeb1, Slug, and Snail, leading to EMT 
reversal. Strikingly, NC attenuated the expression of Nanog, Nestin, Oct-4 and CD44 through HH pathway in breast cancer cells [18]. These reports indicate that NC exerts its tumor suppressive function in breast cancer cells.

\section{Liver cancer}

LC is one of the common malignancies, with HCC as a main subtype [19]. The pathogenesis of HCC and its development process are complex and are related to a variety of signal transduction pathways, including STAT3, SHH, and ERK [20]. One study reported that the NC repressed HCC proliferation though JAK1/STAT3 pathway due to enhancement of apoptosis and increased level of p21 and Bax, and decreased cyclinD1, CDK4 and Bcl-2 in HCC [21]. Similarly, another study also found that NC reduced tumor volume and tumor weight in mice via suppression of ERK, STAT3, and SHH pathways, and regulation of Bcl-2, Bax, CyclinD1, VEGF pathway [20]. These genes are involved in cell proliferation, apoptosis, and tumor angiogenesis in liver cancer [20].

In addition, $\mathrm{Ou}$ et al also found that $\mathrm{NC}$ affected cell proliferation, apoptotic death, and cell cycle by elevation of p53, Bax, caspase-3 and p21, but inhibition of Bcl-2 in liver cancer [1]. Furthermore, a supramolecular formulation of NC has been developed and alleviated hepatotoxicity and improved anticancer activity [22]. Interestingly, NC was found to be a candidate substrate of OCT1 and OCT3 that transfer NC into hepatocytes. Moreover, MATE1 makes NC leave from hepatocytes, while CYPs contributed to NC metabolism such as CYP3A4 and attenuated the hepatic toxicity of NC [23]. TOP1 and TOP2A are key tumor drivers in liver cancer [24], suggesting that TOP1 and TOP2A might be promising targets for treating malignances [25]. One group has shown that the NC exposure attenuated the TOP1 and TOP2A expression levels [26]. Recently, NC was found to suppress the expression of eight genes that are highly expressed in liver cancer [27]. The analogues of nitidine have been synthesized and exhibited extraordinary inhibition of cell proliferation in liver cancer cells [28]. Similarly, supramolecular formulation of NC reduced its toxicity to liver and promoted tumor suppressive activity in liver cancer cells [29]. However, further experiments are necessary to define the molecular mechanism of NC in HCC.

\section{Ovarian cancer}

$O C$ is one type of malignancies in females. The Fas/FasL system is involved in carcinogenesis [30]. Fas can bind to FasL and initiate activation of caspase- 8 and caspase-3 to exert apoptosis [31]. Fas expression was remarkably downregulated in OC patient clinical samples [32, 33]. Therefore, overexpression of Fas could become a potential therapeutic target for OC [34]. NC was found to elevate the level of FADD, caspase- 8 and caspase- 3 in OC cells [34]. Skp2 as an oncoprotein is the key adaptor of the SCF type of E3 ligase [35-37]. NC was reported to inhibit the Skp2 level in OC cells, while Skp2 upregulation abolished NC-induced antitumor property [38]. Thus, suppression of Skp2 expression level by NC might be a useful approach for management of OC patients [38]. There is one study showing that NC blocked cell migratory and invasive activity through downregulation of MMP-2 as well as MMP-9 via suppression of ERK pathway in OC cells [39]. NC induced apoptosis and retarded cell proliferation in OC cells by reduced pAkt and modulation of Bcl-2 family expression [40]. Moreover, NC sensitized OC cells to doxorubicin treatment, leading to a synergistic suppression of proliferation [40].

\section{Renal cancer}

RCC patients with distant metastasis have low five-year survival rate. The underlying molecular mechanisms of renal cancer development and progression are unclear. The higher expression of pAkt is exhibited in RCC, especially in later and metastatic RCC [41, 42]. One study has shown that NC attenuated the cell invasion and metastasis of RCC cells through inhibition of Akt signaling pathway and MMP-2 and MMP-9 [43]. This group further found that NC inhibited phosphorylation of ERK and Akt, upregulated the protein level of p53, Bax, cleavage caspase- 3 and PARP and downregulated Bcl-2 in RCC cells [44]. Without a doubt, the detailed molecular insight into NC-induced antitumor activity in RCC is required to be further investigated.

\section{Glioblastoma}

GBM is one type of aggressive brain malignancy [45]. Due to that GBM is highly proliferative, invasive and chemoresistant, the combined treatments of surgery, chemotherapy, and radiotherapy have not significantly improved the survival rate of GBM patients [46, 47]. Liu et al dissected that NC impaired cell viability and motility of GMB cells via inactivation of pAkt and mTOR [48]. Moreover, NC was reported to increase the expression of cleaved PARP and cleaved caspase 3, and to inhibit pDok2 in GBM cells [49]. Similarly, NC prohibited cell proliferation and colony formation, induced cell cycle arrest at G2/M phase in glioma cells. Notably, NC suppressed GSK3- $\beta$ pathway in glioma cells [49]. Taken together, NC could be a potential anti-glioma agent, which is needed to further explore its antitumor activity. 


\section{Osteosarcoma}

Osteosarcoma is the common bone malignant tumor in young adults and children [50-52]. One study reported that NC prevented the growth of osteosarcoma cells and also stimulated the apoptosis via elevated cleaved forms of caspase- 3 and caspase-9, and increased Bax, and down-regulation of Bcl-2 [53]. Another study revealed that NC inhibited EMT process and suppressed the invasive ability via targeting the Akt/GSK-3 $\beta /$ Snail signaling pathway [54]. Specifically, NC treatment elevated E-cadherin expression and repressed the level of $\mathrm{N}$-cadherin, vimentin, and fibronectn in osteosarcoma cells [54]. Recently, $\mathrm{Xu}$ et al demonstrated that NC impeded proliferation, migration, and invasion, and stimulated apoptosis by inhibition of SIN1 in osteosarcoma cells, indicating that NC might be a useful agent to act as an inhibitor of SIN1 in osteosarcoma [55]. Altogether, NC could be a potential agent for treating osteosarcoma.

\section{Other cancers}

CRC is one of the most commonly diagnosed malignancies. NC was identified to impede the proliferation of CRC cells and induce apoptosis [56]. NC treatment in CRC cells promoted the protein level of Bax, p53, cleaved caspase-3 and -9, and decreased Bcl-2 level and reduced the phosphorylation of ERK, which could lead to cell proliferation inhibition and apoptosis [56]. Elevation of STAT3 expression is observed and associated with metastasis in GC [57]. NC inhibited STAT3 activation, leading to repression of its downstream targets such as cyclin D1, Bcl-xL, and VEGF in GC cells [6]. This finding dissects that NC could be a potent STAT3 inhibitor in gastric cancer to treat this deadly disease. However, further research is necessary to define the mechanism of NC in suppression of growth and metastasis of GC cells.

NC inhibited cell viability via induction of apoptosis by hindering STAT3 pathway in oral cancer cell lines and a tumor xenograft model [58]. NC treatment did not have liver or kidney toxicity in mouse model [58]. This group further identified that NC suppressed Mcl-1 protein level via lysosomedependent degradation in oral squamous cell carcinoma [59]. These reports suggest that NC might be a putative agent against oral cancer [58]. NC exposure exhibited growth inhibition of AML via induction of cell cycle arrest and apoptosis [60]. NC elevated the expression of p27 and Bax, and reduced the level of Cyclin B1, CDK1 and Bcl-2, as well as inactivated PARP in AML cells [60]. Moreover, NC-induced cell growth inhibition in AML cells is partly due to inactivation of the phosphorylation of Akt and ERK [60]. Similarly, NC induced erythroid differentiation and apoptosis in CML via regulation of c-Myc-miRNAs axis [61]. In addition, NC repressed cell proliferation and enhanced apoptotic death through elevation of p53 in NPC cells [62]. Recently, NC was reported to inhibit cell proliferation and invasion through suppression of YAP expression in prostate cancer cells [63]. We believe the tumor suppressive activity of NC will be exhibited in a range of human malignancies.

Table 1. Summary of the functions of nitidine chloride in human cancers.

\begin{tabular}{|c|c|c|c|}
\hline Cancer type & Function & Targets & Reference \\
\hline Breast cancer & $\begin{array}{l}\text { Inhibits growth and induces cell cycle arrest; reduces } \\
\text { migration and invasion; inhibits metastasis. }\end{array}$ & $\begin{array}{l}\text { Downregulates c-Src/FAK; MMP-9, MMP-2, c-Src, FAK, MAPK, RhoA, Rac1, AP, } \\
\text { Bcl-2, smoothened, patched, Gli1, Gli2, Snail, Slug, Zeb1, Nanog, Nestn, Oct-4, and } \\
\text { CD44; upregulates p53, p21, Bax, cleaved caspase-9 and -3, and PARP. }\end{array}$ & {$[3,9,18]$} \\
\hline Liver cancer & $\begin{array}{l}\text { Inhibits cell growth and induces apoptosis and cell } \\
\text { cycle arrest. }\end{array}$ & $\begin{array}{l}\text { Decreases JAK1/STAT3, cyclinD1, CDK4, Bcl-2, ERK, SHH, TOP1 and TOP2A; } \\
\text { upregulates p21, p53, Bax, and caspase-3 and p21. }\end{array}$ & {$[20,21,26]$} \\
\hline Ovarian cancer & $\begin{array}{l}\text { Inhibits proliferation, migration, invasion, and } \\
\text { induced apoptosis. }\end{array}$ & $\begin{array}{l}\text { Downregulates Skp2, MMP-2, MMP-9, ERK, pAkt, Bcl-2. Increases the expression } \\
\text { of Fas, FADD, caspase- } 8 \text { and caspase- } 3 \text {. }\end{array}$ & {$[31,32,38-40]$} \\
\hline Renal cancer & $\begin{array}{l}\text { Suppresses invasion and metastasis; Triggers } \\
\text { apoptosis. }\end{array}$ & $\begin{array}{l}\text { Inhibits AKT signaling pathway, down-regulates MMP-2 and MMP-9. inhibited } \\
\text { phosphorylation of ERK and Akt, upregulates p53, Bax, cleavage caspase-3, and } \\
\text { cleavage PARP, and downregulates Bcl-2, caspase- } 3 \text { and PARP, }\end{array}$ & {$[43,44]$} \\
\hline Glioblastoma & $\begin{array}{l}\text { Inhibits cell viability, migration and invasion, and } \\
\text { induces cell cycle arrest. }\end{array}$ & $\begin{array}{l}\text { Suppresses pAkt, mTOR, pDok2, GSK3-b; increases cleaved PARP and cleaved } \\
\text { caspase } 3 .\end{array}$ & {$[48,49]$} \\
\hline Osteosarcoma & $\begin{array}{l}\text { Inhibits proliferation, migration, invasion and EMT, } \\
\text { induces the apoptosis. }\end{array}$ & $\begin{array}{l}\text { Upregulates cleaved caspase-3, cleaved caspase-9, E-cadherin and Bax. } \\
\text { Downregulates pro-caspase-3, pro-caspase-9, Bcl-2; Akt/GSK-3/Snail, SIN1, } \\
\text { N-cadherin, vimentin, and fibronectn. }\end{array}$ & [53-55] \\
\hline Colorectal cancer & Inhibits proliferation, enhances apoptosis. & Increases Bax, p53, cleaved caspase-3 and -9. Decreases Bcl-2, and pERK. & [56] \\
\hline Gastric cancer & Inhibits angiogenesis and metastasis. & Inhibits STAT3 activation, cyclin D1, Bcl-xL, and VEGF. & [6] \\
\hline Oral cancer & Inhibits cell viability, and enhanced apoptosis. & Downregulates STAT3 signaling pathway, suppresses Mcl-1 level. & {$[58,59]$} \\
\hline $\begin{array}{l}\text { Acute myeloid } \\
\text { leukemia }\end{array}$ & $\begin{array}{l}\text { Inhibits cell growth, induces cell cycle arrest and } \\
\text { apoptosis; }\end{array}$ & Increases p27, Bax; Decreases Cyclin B1, CDK1, Bcl-2, pAkt, and ERK. & [60] \\
\hline $\begin{array}{l}\text { Chronic myeloid } \\
\text { leukemia }\end{array}$ & Induces erythroid differentiation and apoptosis. & Targets c-Myc-miRNAs axis. & {$[61]$} \\
\hline $\begin{array}{l}\text { Nasopharyngeal } \\
\text { carcinoma }\end{array}$ & Inhibits proliferation, and enhances apoptosis. & Upregulates p53. & [62] \\
\hline Prostate cancer & Inhibits cell proliferation and invasion. & Suppresses YAP. & {$[63]$} \\
\hline
\end{tabular}




\section{Conclusions}

NC has been characterized as a potent anti-tumor agent in a wide spectrum of human cancers via various molecular mechanisms (Table 1). In this regard, NC exhibits tumor suppressive functions through targeting numerous signaling pathways. It is noted that NC could have nephrotoxicity due to high-affinity of OCT2 and MATE1 to NC in kidney and have its hepatotoxicity [29, 64]. However, multiple questions should be answered before NC will be used in clinical trial. For example, $\mathrm{NC}$ is absolutely safe for using in human cancer patients? How can NC be delivered to specific organs with tumors? How to improve the NC bio-solubility in human body? What are the detailed and specific molecular mechanisms of NC in different types of human cancers? We believe that answering these questions will promote $\mathrm{NC}$ to be used in clinic trials in the near future.

\section{Abbreviations}

AP: activator protein; AML: acute myeloid leukemia; Bcl-2: B-cell lymphoma 2; CDK4: cyclin-dependent kinase 4; CML: chronic myeloid leukemia; CRC: colorectal cancer; CSC: cancer stem cells; CYPs: cytochrome P450 enzymes; EMT: epithelial mesenchymal transition; ERK: extracellular regulated protein kinases; FADD: Fas-associating protein with a novel death domain; FAK: focal adhesion kinase; FasL: Fas ligand; GBM: Glioblastoma multiforme; GC: gastric cancer; Gli: glioma-associated oncogene homolog; GSK-3 $\beta$ : Glycogen synthase kinase-3 $\beta$; HCC: hepatocellular carcinoma; HIV: human immunodeficiency viruses; HH: hedgehog; JAK1: Janus kinase 1; LC: Liver cancer; MAPK: mitogen-activated protein kinase; MATE1: multidrug and toxin extrusion 1; MCL1: myeloid cell leukemia 1; MMP: Matrix metalloproteinase; mTOR: mammalian target of rapamycin; NC: Nitidine chloride; NPC: nasopharyngeal carcinoma; OC: Ovarian cancer; OCT1: organic cation transporter 1; PARP: poly ADP-ribose polymerase; PDGF: Platelet derived growth factor; RCC: renal cell carcinoma; SCF: Skp1-Cullin1-F-box complex; SHH: sonic hedgehog; Skp2: S-phase kinase associated protein 2; STAT3: signal transducer and activator of transcription 3; TOP1: topoisomerase 1; VEGF: vascular endothelial growth factor; ZEB1: Zinc finger E-box-binding homeobox 1; AP: activator protein; AML: acute myeloid leukemia; Bcl-2: B-cell lymphoma 2; CDK4: cyclin-dependent kinase 4; CML: chronic myeloid leukemia; CRC: colorectal cancer; CSC: cancer stem cells; CYPs: cytochrome P450 enzymes; EMT: epithelial mesenchymal transition; ERK: extracellular regulated protein kinases; FADD: Fas-associating protein with a novel death domain; FAK: focal adhesion kinase; FasL: Fas ligand; GBM: Glioblastoma multiforme; GC: gastric cancer; Gli: glioma-associated oncogene homolog; GSK-3 $\beta$ : Glycogen synthase kinase-3 $\beta$; HCC: hepatocellular carcinoma; HIV: human immunodeficiency viruses; $\mathrm{HH}$ : hedgehog; JAK1: Janus kinase 1; LC: Liver cancer; MAPK: mitogen-activated protein kinase; MATE1: multidrug and toxin extrusion 1; MCL1: myeloid cell leukemia 1; MMP: Matrix metalloproteinase; mTOR: mammalian target of rapamycin; NC: Nitidine chloride; NPC: nasopharyngeal carcinoma; OC: Ovarian cancer; OCT1: organic cation transporter 1; PARP: poly ADP-ribose polymerase; PDGF: Platelet derived growth factor; RCC: renal cell carcinoma; SCF: Skp1-Cullin1-F-box complex; SHH: sonic hedgehog; Skp2: S-phase kinase associated protein 2; STAT3: signal transducer and activator of transcription 3; TOP1: topoisomerase 1; VEGF: vascular endothelial growth factor; ZEB1: Zinc finger E-box-binding homeobox 1 .

\section{Acknowledgments}

This work was supported by the Major project from the Natural Science Foundation of Education Department of Anhui Province (KJ2019ZD27).

\section{Competing Interests}

The authors have declared that no competing interest exists.

\section{References}

1. Khan H, Hadda TB, Touzani R. Diverse Therapeutic Potential of Nitidine, A Comprehensive Review. Curr Drug Metab. 2018; 19: 986-91.

2. Wang Z, Jiang W, Zhang Z, Qian M, Du B. Nitidine chloride inhibits LPS-induced inflammatory cytokines production via MAPK and NF-kappaB pathway in RAW 264.7 cells. J Ethnopharmacol. 2012; 144: 145-50.

3. Pan X, Han H, Wang L, Yang L, Li R, Li Z, et al. Nitidine Chloride inhibits breast cancer cells migration and invasion by suppressing c-Src/FAK associated signaling pathway. Cancer Lett. 2011; 313: 181-91.

4. Bouquet J, Rivaud M, Chevalley S, Deharo E, Jullian V, Valentin A. Biological activities of nitidine, a potential anti-malarial lead compound. Malar J. 2012; 11: 67.

5. Del Poeta M, Chen SF, Von Hoff D, Dykstra CC, Wani MC, Manikumar $\mathrm{G}$, et al. Comparison of in vitro activities of camptothecin and nitidine derivatives against fungal and cancer cells. Antimicrob Agents Chemother. 1999; 43: 2862-8.

6. Chen J, Wang J, Lin L, He L, Wu Y, Zhang L, et al. Inhibition of STAT3 signaling pathway by nitidine chloride suppressed the angiogenesis and growth of human gastric cancer. Mol Cancer Ther. 2012; 11: 277-87.

7. Kovacic P, Ames JR, Lumme P, Elo H, Cox O, Jackson H, et al. Charge transfer-oxy radical mechanism for anti-cancer agents. Anticancer Drug Des. 1986; 1: 197-214.

8. Ponde NF, Zardavas D, Piccart M. Progress in adjuvant systemic therapy for breast cancer. Nature reviews Clinical oncology. 2019; 16: 27-44.

9. Sun M, Zhang N, Wang X, Cai C, Cun J, Li Y, et al. Nitidine chloride induces apoptosis, cell cycle arrest, and synergistic cytotoxicity with doxorubicin in breast cancer cells. Tumour Biol. 2014; 35: 10201-12.

10. Stemmler MP, Eccles RL, Brabletz S, Brabletz T. Non-redundant functions of EMT transcription factors. Nat Cell Biol. 2019; 21: 102-12. 
11. Shibue T, Weinberg RA. EMT, CSCs, and drug resistance: the mechanistic link and clinical implications. Nature reviews Clinical oncology. 2017; 14: 611-29.

12. Dongre A, Weinberg RA. New insights into the mechanisms of epithelial-mesenchymal transition and implications for cancer. Nat Rev Mol Cell Biol. 2019; 20: 69-84.

13. Thiery JP, Acloque H, Huang RY, Nieto MA. Epithelial-mesenchymal transitions in development and disease. Cell. 2009; 139: 871-90.

14. Hollier BG, Evans K, Mani SA. The epithelial-to-mesenchymal transition and cancer stem cells: a coalition against cancer therapies. J Mammary Gland Biol Neoplasia. 2009; 14: 29-43.

15. Lytle NK, Barber AG, Reya T. Stem cell fate in cancer growth, progression and therapy resistance. Nature reviews Cancer. 2018; 18: 669-80.

16. Al-Hajj M, Becker MW, Wicha M, Weissman I, Clarke MF. Therapeutic implications of cancer stem cells. Curr Opin Genet Dev. 2004; 14: 43-7.

17. Shipitsin M, Campbell LL, Argani P, Weremowicz S, Bloushtain-Qimron $\mathrm{N}$, Yao J, et al. Molecular definition of breast tumor heterogeneity. Cancer Cell. 2007; 11: 259-73.

18. Sun M, Zhang N, Wang X, Li Y, Qi W, Zhang H, et al. Hedgehog pathway is involved in nitidine chloride induced inhibition of epithelial-mesenchymal transition and cancer stem cells-like properties in breast cancer cells. Cell Biosci. 2016; 6: 44

19. Ferlay J, Soerjomataram I, Dikshit R, Eser S, Mathers C, Rebelo M, et al. Cancer incidence and mortality worldwide: sources, methods and major patterns in GLOBOCAN 2012. Int J Cancer. 2015; 136: E359-86.

20. Lin J, Shen A, Chen H, Liao J, Xu T, Liu L, et al. Nitidine chloride inhibits hepatic cancer growth via modulation of multiple signaling pathways. BMC Cancer. 2014; 14: 729

21. Liao J, Xu T, Zheng JX, Lin JM, Cai QY, Yu DB, et al. Nitidine chloride inhibits hepatocellular carcinoma cell growth in vivo through the suppression of the JAK1/STAT3 signaling pathway. Int J Mol Med. 2013; 32: 79-84.

22. Siegel RL, Miller KD, Jemal A. Cancer statistics, 2019. CA: a cancer journal for clinicians. 2019; 69: 7-34

23. Li L, Tu M, Yang X, Sun $\mathrm{S}, \mathrm{Wu} X$, Zhou $\mathrm{H}$, et al. The contribution of human OCT1, OCT3, and CYP3A4 to nitidine chloride-induced hepatocellular toxicity. Drug Metab Dispos. 2014; 42: 1227-34.

24. Wang N, Zhu M, Tsao SW, Man K, Zhang Z, Feng Y. MiR-23a-mediated inhibition of topoisomerase 1 expression potentiates cell response to etoposide in human hepatocellular carcinoma. Mol Cancer. 2013; 12: 119.

25. Delgado JL, Hsieh CM, Chan NL, Hiasa H. Topoisomerases as anticancer targets. Biochem J. 2018; 475: 373-98.

26. Liu LM, Xiong DD, Lin P, Yang H, Dang YW, Chen G. DNA topoisomerase 1 and $2 \mathrm{~A}$ function as oncogenes in liver cancer and may be direct targets of nitidine chloride. Int J Oncol. 2018; 53: 1897-912.

27. Liu LM, Lin P, Yang H, Dang YW, Chen G. Gene profiling of HepG2 cells following nitidine chloride treatment: An investigation with microarray and Connectivity Mapping. Oncology reports. 2019; 41: 3244-56.

28. Qin SQ, Li LC, Song JR, Li HY, Li DP. Structurally Simple Phenanthridine Analogues Based on Nitidine and Their Antitumor Activities. Molecules. 2019; 24.

29. Li W, Yin H, Bardelang D, Xiao J, Zheng Y, Wang R. Supramolecular formulation of nitidine chloride can alleviate its hepatotoxicity and improve its anticancer activity. Food and chemical toxicology : an international journal published for the British Industrial Biological Research Association. 2017; 109: 923-9.

30. Rossin A, Miloro G, Hueber AO. TRAIL and FasL Functions in Cancer and Autoimmune Diseases: Towards an Increasing Complexity. Cancers (Basel). 2019; 11.

31. Abrahams VM, Kamsteeg M, Mor G. The Fas/Fas ligand system and cancer: immune privilege and apoptosis. Mol Biotechnol. 2003; 25: 19-30.

32. Ma XY, He FX, Wu SF, Lu YP, Ma D. Expression of survivin in ovarian epithelial carcinoma and its correlation with expression of Fas and FasL. Ai Zheng. 2004; 23: 173-6.

33. Kang HJ, Moon HS, Chung HW. The expression of FAS-associated factor 1 and heat shock protein 70 in ovarian cancer. Obstet Gynecol Sci. 2014; 57: 281-90.

34. Chen S, Yang L, Feng J. Nitidine chloride inhibits proliferation and induces apoptosis in ovarian cancer cells by activating the Fas signalling pathway. J Pharm Pharmacol. 2018; 70: 778-86.

35. Chan $\mathrm{CH}$, Morrow JK, Zhang S, Lin HK. Skp2: a dream target in the coming age of cancer therapy. Cell Cycle. 2014; 13: 679-80.

36. Fu HC, Yang YC, Chen YJ, Lin H, Ou YC, Chien CC, et al. Increased expression of SKP2 is an independent predictor of locoregional recurrence in cervical cancer via promoting DNA-damage response after irradiation. Oncotarget. 2016; 7: 44047-61.

37. Wang Z, Liu P, Inuzuka H, Wei W. Roles of F-box proteins in cancer. Nat Rev Cancer. 2014; 14: 233-47.
38. Mou H, Guo P, Li X, Zhang C, Jiang J, Wang L, et al. Nitidine chloride inhibited the expression of $S$ phase kinase-associated protein 2 in ovarian cancer cells. Cell Cycle. 2017; 16: 1366-75.

39. Sun X, Lin L, Chen Y, Liu T, Liu R, Wang Z, et al. Nitidine chloride inhibits ovarian cancer cell migration and invasion by suppressing MMP-2/9 production via the ERK signaling pathway. Mol Med Rep. 2016; 13: 3161-8.

40. Ding F, Liu T, Yu N, Li S, Zhang X, Zheng G, et al. Nitidine chloride inhibits proliferation, induces apoptosis via the Akt pathway and exhibits a synergistic effect with doxorubicin in ovarian cancer cells. Mol Med Rep. 2016; 14: 2853-9.

41. Horiguchi A, Oya M, Uchida A, Marumo K, Murai M. Elevated Akt activation and its impact on clinicopathological features of renal cell carcinoma. J Urol. 2003; 169: 710-3.

42. Kuroda K, Horiguchi A, Sumitomo M, Asano T, Ito K, Hayakawa M, et al. Activated Akt prevents antitumor activity of gefitinib in renal cancer cells. Urology. 2009; 74: 209-15.

43. Fang Z, Tang Y, Jiao W, Xing Z, Guo Z, Wang W, et al. Nitidine chloride inhibits renal cancer cell metastasis via suppressing AKT signaling pathway. Food Chem Toxicol. 2013; 60: 246-51.

44. Fang Z, Tang Y, Jiao W, Xing Z, Guo Z, Wang W, et al. Nitidine chloride induces apoptosis and inhibits tumor cell proliferation via suppressing ERK signaling pathway in renal cancer. Food Chem Toxicol. 2014; 66: 210-6.

45. Ricard D, Idbaih A, Ducray F, Lahutte M, Hoang-Xuan K, Delattre JY. Primary brain tumours in adults. Lancet. 2012; 379: 1984-96.

46. Stupp R, Brada M, van den Bent MJ, Tonn JC, Pentheroudakis G, Group EGW. High-grade glioma: ESMO Clinical Practice Guidelines for diagnosis, treatment and follow-up. Ann Oncol. 2014; 25 Suppl 3: iii93-101

47. Van Meir EG, Hadjipanayis CG, Norden AD, Shu HK, Wen PY, Olson JJ. Exciting new advances in neuro-oncology: the avenue to a cure for malignant glioma. CA Cancer J Clin. 2010; 60: 166-93.

48. Liu M, Wang J, Qi Q, Huang B, Chen A, Li X, et al. Nitidine chloride inhibits the malignant behavior of human glioblastoma cells by targeting the PI3K/AKT/mTOR signaling pathway. Oncol Rep. 2016; 36: 2160-8.

49. Deshpande RP, Babu PP. pDok2, caspase 3 dependent glioma cell growth arrest by nitidine chloride. Pharmacol Rep. 2018; 70: 48-54.

50. Huh WW, Holsinger FC, Levy A, Palla FS, Anderson PM. Osteosarcoma of the jaw in children and young adults. Head Neck. 2012; 34: 981-4.

51. Mirabello L, Troisi RJ, Savage SA. Osteosarcoma incidence and survival rates from 1973 to 2004: data from the Surveillance, Epidemiology, and End Results Program. Cancer. 2009; 115: 1531-43.

52. Ottaviani G, Jaffe N. The epidemiology of osteosarcoma. Cancer Treat Res. 2009; 152: 3-13

53. $\mathrm{Xu} \mathrm{Q}, \mathrm{Li} \mathrm{ZX}$, Ye ZM. Nitidine chloride-induced apoptosis of human osteosarcoma cells and its mechanism. Nan Fang Yi Ke Da Xue Xue Bao. 2011; 31: 361-4.

54. Cheng Z, Guo Y, Yang Y, Kan J, Dai S, Helian M, et al. Nitidine chloride suppresses epithelial-to-mesenchymal transition in osteosarcoma cell migration and invasion through Akt/GSK-3beta/Snail signaling pathway. Oncol Rep. 2016; 36: 1023-9.

55. Xu H, Cao T, Zhang X, Shi Y, Zhang Q, Chai S, et al. Nitidine Chloride Inhibits SIN1 Expression in Osteosarcoma Cells. Mol Ther Oncolytics. 2019; 12: 224-34.

56. Zhai $\mathrm{H}, \mathrm{Hu} \mathrm{S}$, Liu T, Wang F, Wang $\mathrm{X}, \mathrm{Wu}$ G, et al. Nitidine chloride inhibits proliferation and induces apoptosis in colorectal cancer cells by suppressing the ERK signaling pathway. Mol Med Rep. 2016; 13: 2536-42.

57. Cafferkey C, Chau I. Novel STAT 3 inhibitors for treating gastric cancer. Expert Opin Investig Drugs. 2016; 25: 1023-31.

58. Kim LH, Khadka S, Shin JA, Jung JY, Ryu MH, Yu HJ, et al. Nitidine chloride acts as an apoptosis inducer in human oral cancer cells and a nude mouse xenograft model via inhibition of STAT3. Oncotarget. 2017; 8: 91306-15.

59. Yang IH, Jung W, Kim LH, Shin JA, Cho NP, Hong SD, et al. Nitidine chloride represses Mcl-1 protein via lysosomal degradation in oral squamous cell carcinoma. J Oral Pathol Med. 2018; 47: 823-9.

60. Li P, Yan S, Dong X, Li Z, Qiu Y, Ji C, et al. Cell Cycle Arrest and Apoptosis Induction Activity of Nitidine Chloride on Acute Myeloid Leukemia Cells. Med Chem. 2018; 14: 60-6.

61. Liu N, Li P, Zang S, Liu O, Ma D, Sun X, et al. Novel agent nitidine chloride induces erythroid differentiation and apoptosis in CML cells through c-Myc-miRNAs axis. PLoS One. 2015; 10: e0116880.

62. Kang M, Ou H, Wang R, Liu W, Tang A. The effect of nitidine chloride on the proliferation and apoptosis of nasopharyngeal carcinoma cells. Journal of BUON 2014; 19: 130-6. 
63. Shi Y, Cao T, Sun Y, Xia J, Wang P, Ma J. Nitidine Chloride inhibits cell proliferation and invasion via downregulation of YAP expression in prostate cancer cells. Am J Transl Res. 2019; 11: 709-20.

64. Li LP, Song FF, Weng YY, Yang X, Wang K, Lei HM, et al. Role of OCT2 and MATE1 in renal disposition and toxicity of nitidine chloride. Br J Pharmacol. 2016; 173: 2543-54. 